\title{
Application of Recellularized Non-Woven Materials from Collagen-Enriched Polylactide for Creation of Tissue-Engineered Diaphragm Constructs
}

DOI: $10.17691 / \mathrm{stm} 2019.11 .2 .05$

Received June 20, 2018

E.V. Kuevda, PhD, Researcher, Laboratory of Fundamental Research in the Field of Regenerative Medicine ${ }^{1}$;

E.A. Gubareva, PhD, Head of the Laboratory of Fundamental Research in the Field of Regenerative Medicine ${ }^{1}$;

T.E. Grigoriev, PhD, Head of the Department of Nanobiomaterials and Structures, Kurchatov Complex of NBICS-Technologies2;

S.V. Krasheninnikov, Researcher, Laboratory of Polymer Materials, Kurchatov Complex of NBICS-Technologies;

A.A. Verevkin, PhD Student, Laboratory of Fundamental Research in the Field of Regenerative Medicine';

K.I. Lukanina, PhD, Researcher, Department of Nanobiomaterials and Structures, Kurchatov Complex

of NBICS-Technologies';

D.P. Puzanov, MD, PhD, Researcher, Laboratory of Fundamental Research in the Field of Regenerative Medicine ${ }^{1}$;

I.Kh. Yegiyev, Medical Resident, Laboratory of Fundamental Research in the Field of Regenerative Medicine";

A.L. Vasiliev, PhD, Head of the Laboratory, Kurchatov Complex of NBICS-Technologies;;

S.N. Chvalun, DSc, Deputy Director for Scientific Work, Kurchatov Complex of NBICS-Technologies ${ }^{2}$

${ }^{1}$ Kuban State Medical University, 4 M. Sedin St., Krasnodar, 350063, Russia;

${ }^{2}$ National Research Center "Kurchatov Institute", 1 Akademika Kurchatova Square, Moscow, 123182, Russia

The aim of the investigation was to study biocompatibility and biomechanical properties of recellularized non-woven materials based on collagen-filled polylactide under in vitro and in vivo conditions and to assess the potential of their use for diaphragm defect replacement in the experiment on small laboratory animals.

Materials and Methods. Non-woven materials were obtained by electroforming of polylactide in a tight box with subsequent specimen vacuumization. $9 \%$ aqueous solution of polylactide was prepared in the solvent mixture: chemically pure chloroform with $10 \%$ addition of chemically pure ethanol. The microfibrous material was enriched with collagen in a weight concentration of 0.1 and $0.5 \%$ with subsequent freezing at $-40^{\circ}$ or in liquid nitrogen. The structure of non-woven polylactide matrices was assessed by a scanning electron microscopy while mechanical properties were evaluated during cyclic tests for strength and fatigue properties using tensile-testing machine. The fabricated non-woven matrices were recellularized by mesenchymal stromal cells under static conditions. The quality of the fabricated tissue-engineered constructs was evaluated morphologically by routine histological investigations. The viability of the cells on the matrices and the cytotoxic properties of the matrices were determined using XTT tests. The least toxic specimens were orthotopically implanted into the rats with subsequent morphological examinations.

Results. The morphological analysis and viability study of the cells on the matrices of those specimens which underwent freezing in liquid nitrogen during forming were found to be inexpedient for orthotopic transplantation. The specimens frozen in the refrigeration unit demonstrated permissible cytotoxicity levels and were implanted. The morphological examination after explantation of the given specimen groups did not reveal significant damage to the structure and inflammatory changes though the signs of the marked adhesive process in the implantation area were evident.

Conclusion. In spite of the ability of the non-woven matrices to stimulate fibrinogenesis, absence of cytotoxic properties and evident reactions of transplant rejection allow the non-woven polylactide matrices undergone freezing in the refrigeration unit at $-40^{\circ}$ during the forming process to be considered promising for creation of tissue-engineered constructs of a diaphragm. cells.

Key words: tissue engineering; non-woven material; diaphragm; polylactide non-woven matrices; transplantation; mesenchymal stromal

Corresponding author: Elena V. Kuevda, e-mail: elenakuevda@yandex.ru 


\section{Introduction}

Congenital and acquired malformations of various organs, including the diaphragm, remain a serious problem with a high level of lethality [1]. Though the arsenal of therapeutic and surgical treatment methods has recently enlarged, the most serious cases still have fatal outcomes. Regenerative medicine offers new methods of treating congenital and acquired pathologies creating tissue-engineered constructs (TEC) based on biological and/or synthetic matrices and a cell component [2]. For example, presently, there are various open and minimally invasive ways of plastic defect closure including the diaphragm [3].

The majority of plastic operations are performed using synthetic materials based on polytetrafluoroethylene, polyethylene, and polypropylene (AlloDerm, GoreTex, Marlex) which lack bioresorbability and, therefore, promote the recurrence of the pathological process $[1,4]$. Non-woven biodegradable polycaprolactonebased materials are considered by many authors the most perspective for replacing the defects of the skin, bone tissue, small vessels, and diaphragm [5-8]. At the same time, polylactide, being an inexpensive material, possesses a required strength (greater than polycaprolactone), biocompatibility, biodegradability without toxic metabolites making it rather promising for fabricating implants and non-woven materials necessary in regenerative medicine. Unfortunately, much less attention is paid to the study of the biocompatibility of polylactide-based TECs compared to the polycaprolactone-based matrices [9].

There are data on a successful application of combined synthetic non-woven collagen-filled materials for the replacement of vast (more than 70\%) defects of the diaphragm in the experiment even without the addition of a cellular component [8]. However, at present, the non-woven matrices are often used as carriers for mesenchymal stromal cells (MSC) during the creation of synthetic prostheses in order to improve biocompatibility and decrease immunogenicity of the implanted material $[10,11]$.

The majority of the investigations performed [8] are focused on the study of biomechanical and bioresorptive properties of the non-woven materials, the choice of optimal way of implant population in vitro. And a cellular component is considered as an additional factor promoting the replacement of the prosthetic area by the autologous connective tissue of the recipient [7] without adequate reconstruction of the anatomical and physiological properties of the native organ. Implantation of recellularized synthetic matrix into the animal organism and subsequent examination of the efficiency of the tissue defect replacement, cell growth, and MSC proliferation on the synthetic matrix as a stage of TEC creation is an interesting but insufficiently studied task [2]. Selection of the polymer, a carrier of a cell component, as well as optimal conditions of material forming is also an important constituent of the problem of creating TEC based on the non-woven matrices.

The aim of the investigation was to study the properties of recellularized non-woven materials based on collagen-filled polylactide under in vitro and in vivo conditions and to assess the possibility of using these materials for diaphragm defect replacement in the experiment on small laboratory animals.

\section{Materials and Methods}

Fabrication and description of matrices, preparation to recellularization and its conduction. Polylactide-based non-woven materials were obtained by electroforming in a tight box at room temperature with subsequent vacuumization of the specimens at $1 \mathrm{mbar}$ evacuation till the complete solvent removal. $9 \%$ aqueous solution of polylactide (4032D; Nature Works, USA) was preliminarily prepared in the solvent mixture: chemically pure chloroform with $10 \%$ addition of chemically pure ethanol. The microfibrous material was obtained on the mandrel $12.0 \pm 0.5 \mathrm{~cm}$ in diameter, rotating at $40 \mathrm{rpm}$, and the applied voltage was $15.0 \pm 1.0 \mathrm{kV}$. The process flow rate was $5 \mathrm{ml} / \mathrm{h}$. The obtained fibrous matrix was $0.40 \pm 0.05 \mathrm{~mm}$ thick. The fiber diameter ranged from 4.5 to $5.5 \mu$ with an average value of $5.0 \mu$. The packing density in the middle area was $10 \%$.

To enrich collagen matrices, improve their adhesive properties and biocompatibility, we used collagen in 0.1 and $0.5 \%$ weight concentration with subsequent freezing in a refrigeration unit at $-40^{\circ}$ or in liquid nitrogen. Then the specimens underwent lyophilisation in Alpha 2-4 LSCplus freeze-dryer (Martin Christ, Germany) for $24 \mathrm{~h}$. Thus, four groups of matrices have been fabricated for subsequent recellularization under in vitro conditions. Experimental group 1 included polylactide specimens containing $0.1 \%$ of collagen which underwent freezing in liquid nitrogen; group 2 was comprised of the specimens with the same content of collagen but frozen at $-40^{\circ}$. Groups 3 and 4 consisted of non-woven polylactide matrices containing $0.5 \%$ of collagen and frozen in liquid nitrogen and at $-40^{\circ}$, respectively. A nonwoven material consisting of collagen-free polylactide was used as a control. The structural and mechanical properties of the obtained non-woven materials were assessed by a scanning electron microscopy using Helios NanoLab 450 S system (Thermo Fisher Scientific, USA) and by cyclic mechanical tests for strength and fatigue characteristics using Instron 5965 tensile testing machine (ITW, USA).

Before recellularization, the specimen were sterilized with UV irradiation for $30 \mathrm{~min}$, immersed in $70^{\circ}$ ethanol for $10 \mathrm{~min}$, washed three times in the solution of phosphate buffer for 15 min (Thermo Fisher Scientific, USA), and placed in the DMEM/F12 culture medium (PanEco, Russia) for 20 min. Using DERMO-PUNCH device (Sterylab, Italy) for skin biopsy, specimens of non-woven materials $5 \mathrm{~mm}$ in diameter were obtained 
to investigate in vitro the viability of the cells on the matrices and cytotoxic properties of the matrices themselves (XTT tests).

Specimens measuring $1.5 \times 2 \mathrm{~cm}$, that is about $70 \%$ of the left diaphragm cupula, were chosen for orthotopic transplantation. Static recellularization of these specimens was performed in 6-well plates for 16 days in a $\mathrm{CO}_{2}$ incubator under standard conditions. Matrices displaying the least cytotoxic properties were chosen for implantation. The efficiency of recellularization of the remained specimens was evaluated during morphological examination by staining with hematoxylin and eosin according to the standard protocol.

Rat bone marrow-derived MSCs of the fourth passage were used for the experiments on non-woven matrix population. Cell lineage was verified by immunotyping, assessment of superficial antigen expression, and by the capability of directional differentiation in three cell lines using specialized StemPro media (Thermo Fisher Scientific, USA) with subsequent qualitative detection.

The study of viability of the cells on matrices. Colorimetric XTT test with the following quantitative counting was chosen to determine the percentage of viable cells on the matrices and the cytotoxic index. The process solution of the XTT reagent was prepared according to the manufacturer instructions (Cell proliferation assay XTT; AphliChem $\mathrm{GmbH}$, Germany). Recellularization of the experimental and control samples was performed in 96-well plates by a static method, $130 \mu \mathrm{l}$ cell suspension containing 20,000 cells was added to each well. The cell suspension was applied to the specimen central zone and uniformly distributed over the entire surface. The total time of incubation was $72 \mathrm{~h}$. Then $100 \mu \mathrm{l}$ of the XTT working solution was added followed by a further $3 \mathrm{~h}$ incubation. The optical density was measured by means of FilterMax F5 reader (Molecular Devices, USA) at $450 \mathrm{~nm}$ working wavelength (620 nm reference wavelength). The quantitative values of the cell viability were calculated as a ratio of the difference of the optical density values in the experimental wells and the wells with the medium to the optical density of the wells containing the control cells [12].

Implantation and explantation of recellularized non-woven matrices. The TEC diaphragm based on MSC recellularized non-woven matrices enriched with collagen $(n=25)$ was orthotopically transplanted to 5 Wistar male rats weighing $240 \pm 20 \mathrm{~g}$. The experiments on the animals were conducted in the Laboratory of Basic Research in Regenerative Medicine in Kuban State Medical University in compliance with the Guide for the Care and Use of Laboratory Animals (National Research Council, 2011). The study protocols were designed according to the European Convention for the Protection of Vertebrate Animals used for Experimental and Other Scientific Purposes (Strasbourg, 2006) and approved by the Ethics Committee of the Kuban State Medical University.

The operative interventions on animals were done under general anesthesia, the protocol of which was worked out together with the colleagues from the Karolinska Institute (Stockholm, Sweden). It implied intramuscular injection of $5 \mathrm{ME}$ of $2 \%$ Xylazine solution (Intervet, Netherlands) in combination with $3 \mathrm{ME}$ Zoletil 100 (Virbac, France) and subcutaneous introduction of $5 \mathrm{ME}$ of $0.1 \%$ Atropine sulfate solution (Pilot Plant "GNCLS", Ukraine). According to the protocol, rat tracheas were intubated with a polyethylene catheter $2.42 \mathrm{~mm}$ in diameter followed by the connection to the artificial pulmonary ventilation device Inspira ventilator (Harvard Apparatus; USA) for small animals with a specified respiration rate of 100 per minute. The animals were fixed in a supine position for oblique paracostal laparotomy, left approach. About $70 \%$ of the left diaphragm cupula was ablated and replaced with the non-woven material populated with the rat MSCs. Implants were fixed to the pericostal part of the diaphragm by continuous sutures using monofilament polypropylene suture (size 6-0). The laparotomy wound was closed in layers using a continuous suture with a monofilament polypropylene suture (size 4-0) (all sutures were Prolene; Ethicon, USA). The total length of the operative intervention made $50 \mathrm{~min}$. In the postoperative period, in case of wound suppuration, infectious complications were prevented using $2.5 \%$ solution of Baytril preparation (VNIIZG, Russia) at a dose of $0.2 \mathrm{ml} /$ $\mathrm{kg}$ animal body mass, immunosuppressive therapy was not administered.

21 days after the implantation, the non-woven recellularized grafts were explanted after the intraperitoneal introduction of the lethal dose of barbiturates $(150 \mathrm{mg} / \mathrm{kg})$. A left paracostal approach was used during surgery, the process of explantation was accompanied by macrophotography. Blood was washed off the specimens in a sterile solution of the phosphate buffer. Then specimens were placed in $10 \%$ solution of buffered formalin for histological investigations.

Statistical data processing was performed using GraphPad Prism v. 6.04 software (reference: www. graphpad.com). The results obtained were presented as the mean (M) and standard deviation (SD). The probability of the possible error was determined using the Student's t-test table for paired samples. MannWhitney test was used for the samples from compared populations different from normal distribution. The differences were considered significant at $p<0.05$.

\section{Results}

The description of the structure and methods of nonwoven specimen treatment is presented in Table 1, their mechanical parameters are shown in Table 2.

The cell culture used for recellularization of the nonwoven matrices demonstrated its ability to adhesion and colony formation growing on the cultural plastic. The cells had $\mathrm{CD}^{\circ} 0^{+}, \mathrm{CD}_{105^{+}}, \mathrm{CD} 34^{-}, \mathrm{CD} 45^{-}$phenotype, after the induced differentiation they were positively stained 
Table 1

Description of the structure and methods of non-woven material treatment

\begin{tabular}{|c|c|c|c|c|c|}
\hline $\begin{array}{l}\text { Specimen } \\
\text { groups }\end{array}$ & $\begin{array}{l}\text { Thickness } \\
\text { of the fibrous matrix, } \\
\text { mm/packing density (\%) }\end{array}$ & $\begin{array}{c}\text { Fiber } \\
\text { diameter }(\mu \mathrm{m})\end{array}$ & $\begin{array}{l}\text { Concentration } \\
\text { of filler solution } \\
\text { (collagen) }(\%)\end{array}$ & $\begin{array}{l}\text { Speed } \\
\text { of freezing } \\
\left({ }^{\circ} \mathrm{C} / \mathrm{min}\right)\end{array}$ & $\begin{array}{l}\text { The degree } \\
\text { of filling the specimen } \\
\text { with collagen (\%) }\end{array}$ \\
\hline 1 & \multirow{4}{*}{$0.40 \pm 0.05 / 10.0 \pm 1.0$} & \multirow{4}{*}{$5.0 \pm 0.5$} & 0.1 & $10.0^{* *}$ & 6.8 \\
\hline 2 & & & 0.1 & $0.05^{\star}$ & 4.3 \\
\hline 3 & & & 0.5 & $10.0^{* *}$ & 16.0 \\
\hline 4 & & & 0.5 & $0.05^{*}$ & 20.7 \\
\hline
\end{tabular}

${ }^{*} 0.05^{\circ} \mathrm{C} / \mathrm{min}$ - freezing in a refrigeration unit; ${ }^{* *} 10.0^{\circ} \mathrm{C} / \mathrm{min}$ - freezing in liquid nitrogen.

Table 2

Mechanical characteristics of the non-woven material specimens ( $\mathrm{MPa})$

\begin{tabular}{ccccc}
\hline \multirow{2}{*}{$\begin{array}{c}\text { Type } \\
\text { of freezing }\end{array}$} & \multicolumn{2}{c}{$\begin{array}{c}\text { Young } \\
\text { modulus }\end{array}$} & Strength & \multicolumn{2}{c}{$\begin{array}{c}\text { Young } \\
\text { modulus }\end{array}$} & Strength \\
\hline $0.05^{\circ} \mathrm{C} / \mathrm{min}$ & $434.0 \pm 25.0$ & $13.2 \pm 0.8$ & $508.0 \pm 31.0$ & $20.7 \pm 0.9$ \\
\hline $10.0^{\circ} \mathrm{C} / \mathrm{min}$ & $338.0 \pm 21.0$ & $11.3 \pm 0.7$ & $529.0 \pm 34.0$ & $21.8 \pm 1.1$ \\
\hline
\end{tabular}

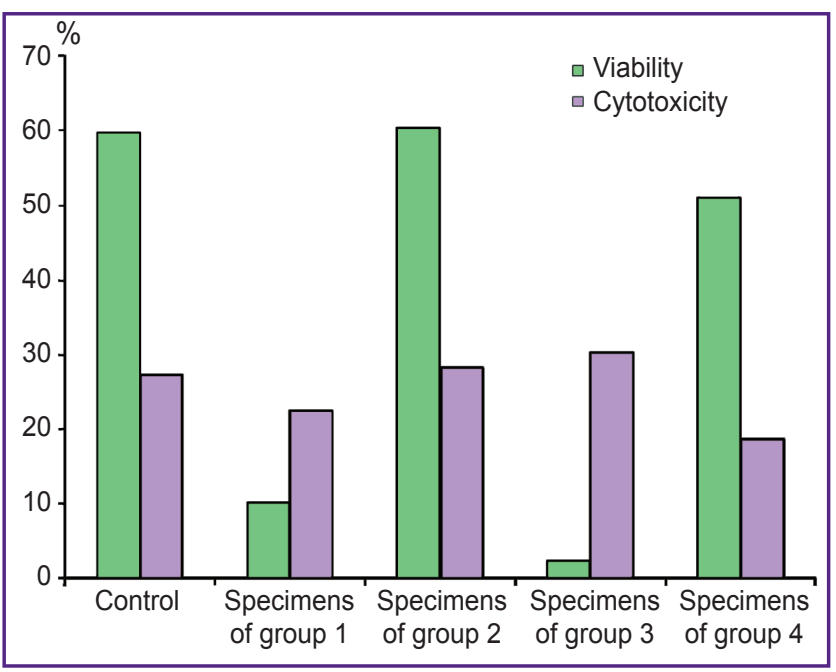

Figure 1. Indices of viability and cytotoxicity of the nonwoven synthetic matrix based on collagen-enriched polylactide with recellularization with bone marrowderived rat mesenchymal stromal cells

with Oil Red O, toluidine blue, alizarin red, that validates the differentiation in adipogenous, chondrogenous, and osteogenous directions, respectively.

According to the data of the XTT test, the viability of the cells after recellularization of the specimens from the control group after the static population by MSCs was $59.76 \%$, the cytotoxicity $-27.23 \%$. Among the specimens of the experimental groups, the matrices of group 2 and 4 frozen in the refrigeration unit possessed the least cytotoxic properties. The same groups of specimens were characterized by the highest viability of the cells on the matrices (Figure 1).
It was decided to examine the histological structure of the specimens with high values of cytotoxicity (group 1 and 3) to establish the reasons for such high indices. Staining with hematoxylin and eosin showed heterogeneity of the structure on the frontal sections, stratification of the material, and fiber constrictions on the sagittal sections; cells were absent (Figure 2). Scanning electron microscopy of the non-woven matrix specimens from these groups performed before MSC population showed marked interfibrillar connections in the form of membranes and constrictions (Figure 3).

During explantation, it was found that tissueengineered grafts obtained by recellularization of the non-woven materials were integrated into the surrounding tissues with the implantation area having a paler color compared to the normal diaphragm tissue. Explanted grafts with the highest cell viability were noted to have a high degree of adhesion of the implanted materials to the surrounding tissues. Thus, the specimen from group 2 fused to the stomach fundus and diaphragmatic surface of the liver, the specimen from group 4 to the diaphragmatic surface of the liver, the control specimen was seen to be tightly adhered to

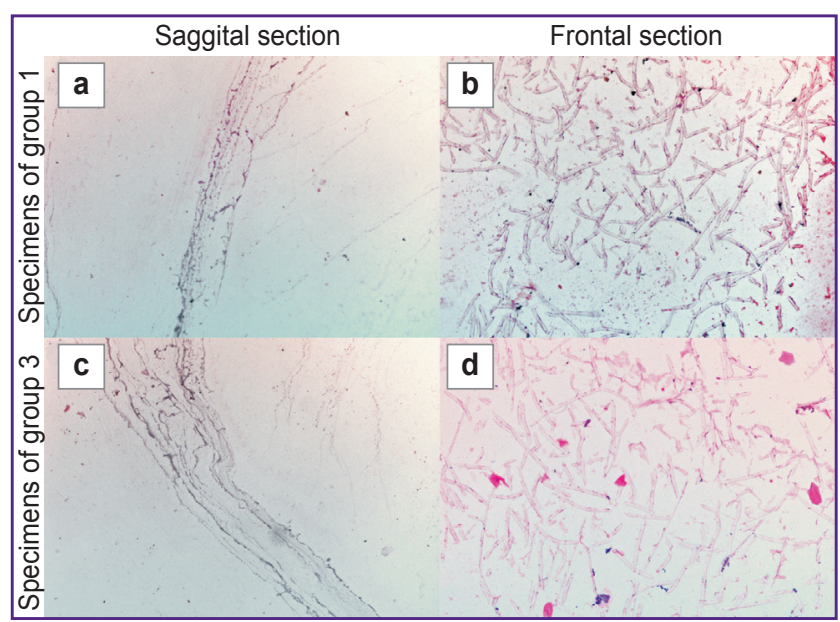

Figure 2. Morphological in vitro examination of recellularized non-woven matrices:

saggital (a) and frontal (b) sections of specimens from group 1; saggital (c) and frontal (d) sections of specimens from group 3; staining with hematoxylin and eosin; $x 100$ 
Figure 3. Electron micrographs of the nonwoven matrices based on collagen-filled polylactide:

(a) fibers connected by membranes, degree of filling - 20.7\%; (b) fibers connected by constriction, degree of filling $-6.8 \% ; \times 2500$
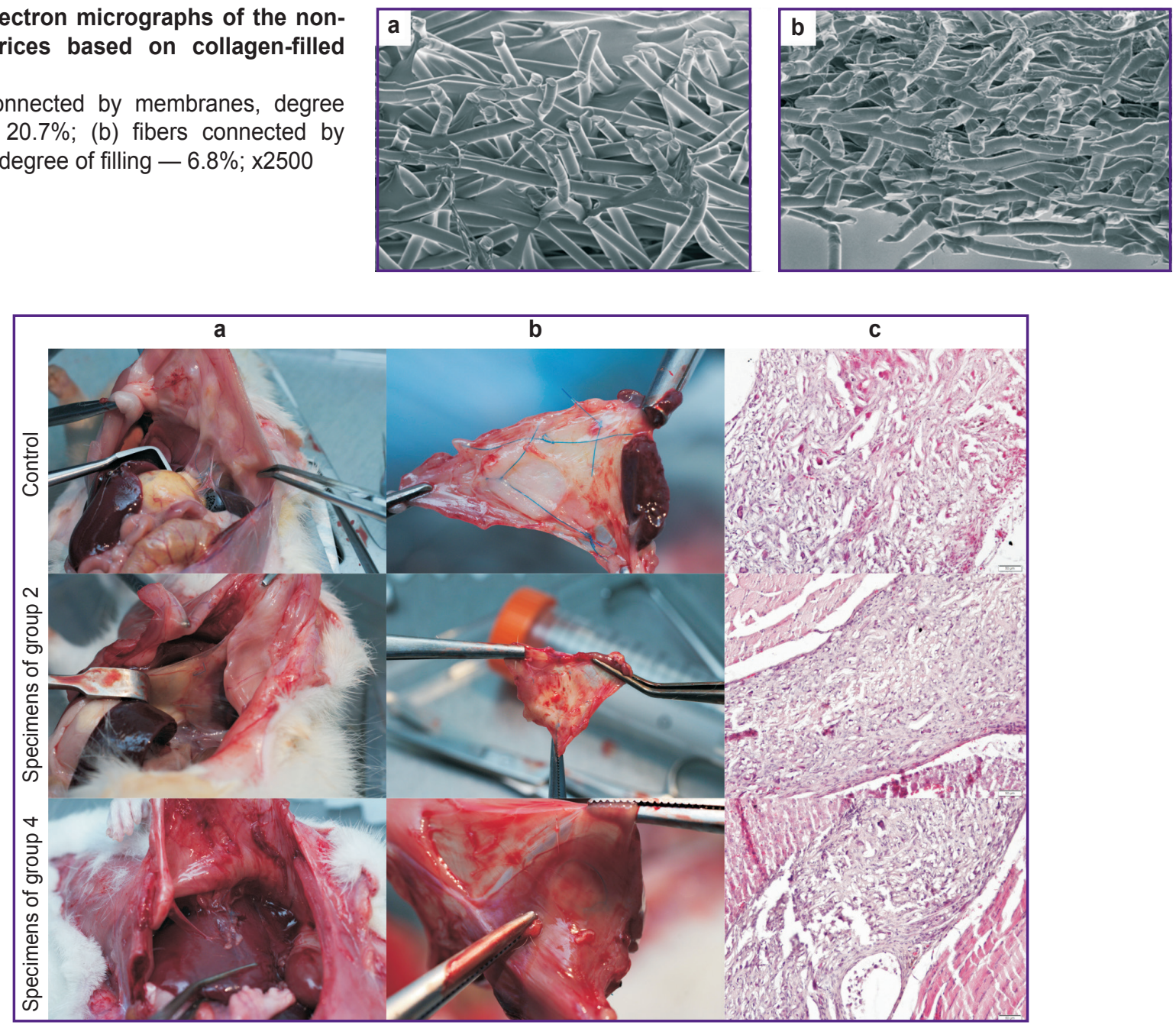

Figure 4. Macro photography and histological examination of recellularized non-woven matrices 21 days after implantation:

(a) adhesive process in the implantation area; (b) implanted specimen; (c) morphologic examination of the explanted specimens; staining with hematoxylin and eosin; x200

the stomach fundus (Figure 4 (a), (b)). Ablation of the left diaphragm cupula was done after blunt dissection of adhesions.

The morphological examination of the explanted grafts stained with hematoxylin and eosin allowed us to assess the general picture of cell infiltration, predominantly leukocytic. At the same time, no signs of evident inflammatory changes were found, the implanted TECs were not limited by the capsule from the surrounding tissues. The explants preserved a porous structure not typical for the native diaphragmatic tissue. Significant morphological differences between the specimens of the control group and experimental groups 2 and 4 have not been noted (Figure 4 (c)).

\section{Discussion}

The non-woven materials, their recellulalization, and the application of the fabricated grafts for native organ replacement are considered to be a promising way of solving the tasks on adequately functioning TECs. It has been shown that an important step in creation of synthetic prostheses is the process of electroforming with the choice of the optimal micropore diameter (within the range of 20-80 $\mu \mathrm{m}$ ), calculation of the porous material percentage, selection of matrix thickness, and fiber diameter [13]. Of equal importance are the method of the non-woven material population and potential ways of improving matrix functional properties using additional treatment (for example, enrichment with collagen) $[14,15]$. The choice of the method of synthetic material population is known to depend on electroforming conditions determining the structural and mechanical properties of the created matrices and the required parameters $[7,16]$.

Though some investigators criticize the static method of recellularization [13], it was this particular method that we have chosen making some modifications regarding the application of cell suspension (by drops to the middle 
of the specimen with subsequent distribution over the surface). Our choice was inspired by the successful experience of using the static method of diaphragm repopulation by various cell lines [3, 17]. We have also decided to apply an additional collagen coating on the synthetic materials in order to improve biocompatibility. In the course of the experiment, it was established that the materials subjected to freezing in liquid nitrogen demonstrated a lower ability to maintain growth and proliferation of the cells irrespective of the collagen percentage $(10.08 \%$ of viable cells for the specimens of group 1 and $2.31 \%$ for group 3 ). Low viability of MSCs on the specimens of groups 1 and 3 is likely to be caused by the relief matrix morphology and presence of collagen constrictions induced by rapid freezing in nitrogen [18]. The histological examination showed that these specimens did not sufficiently preserve the structural characteristics of the matrices and confirmed the inexpediency of orthotopic transplantations using the TECs fabricated by this method.

Specimens exposed to low temperatures in the refrigeration unit possessed the cytotoxic properties comparable with the control. The cytotoxic index of the specimens from group 2 was $28.21 \%$ and that of the control $27.23 \%$. The addition of $0.5 \%$ of collagen during the electroforming of the specimens from group 4 contributed to the decrease of the cytotoxic index by $8.72 \%$ relative to the control.

There was a negative fact revealed in the course of our experiment: during explantation of the specimens of the control group and experimental groups 2 and 4, marked adhesive processes in the abdominal cavity involving the diaphragmatic surface of the liver and gastric fundus were detected 21 days after the implantation. At the same time, there were no evident inflammatory changes and rejection of the implanted TEC.

\section{Conclusion}

Additional improvement of biomechanical properties of non-woven synthetic matrices (polylactide, in particular) by the introduction of collagen into their composition is able to increase the viability of the cells on the matrices if optimal conditions of temperature treatment are chosen at the stage of forming (freezing in the refrigeration unit). Besides, recellularized synthetic matrices, in contrast to decellularized, demonstrate the greater ability to trigger adhesive processes in the abdominal cavity [3] in case other conditions of matrix repopulation, operative intervention, and postoperative management of the animals are equal. The indicated features of the non-woven synthetic materials dictate the necessity of searching additional methods of matrix treatment to prevent excessive adhesion to the adjacent tissues.

Study funding. The work was supported by the grant of the Russian Scientific Foundation (project No.17-1301376).
Conflicts of interest. The authors have no conflicts of interest to disclose.

\section{References}

1. De Coppi P., Deprest J. Regenerative medicine solutions in congenital diaphragmatic hernia, seminars in pediatric surgery. Semin Pediatr Surg 2017; 26(3): 171-177, https://doi.org/10.1053/j.sempedsurg.2017.04.009.

2. De Coppi P. Regenerative medicine for congenital malformations. J Pediatr Surg 2013; 48(2): 273-280, https:// doi.org/10.1016/j.jpedsurg.2012.11.005.

3. Gubareva E.A., Sjöqvist S., Gilevich I.V., Sotnichenko A.S., Kuevda E.V., Lim M.L., Feliu N., Lemon G., Danilenko K.A., Nakokhov R.Z., Gumenyuk I.S., Grigoriev T.E., Krasheninnikov S.V., Pokhotko A.G., Basov A.A., Dzhimak S.S., Gustafsson Y., Bautista G., Beltrán Rodríguez A., Pokrovsky V.M., Jungebluth P., Chvalun S.N., Holterman M.J., Taylor D.A., Macchiarini P. Orthotopic transplantation of a tissue engineered diaphragm in rats. Biomaterials 2016; 77: 320-335, https://doi.org/10.1016/j. biomaterials.2015.11.020.

4. Romao R.L., Nasr A., Chiu P.P., Langer J.C. What is the best prosthetic material for patch repair of congenital diaphragmatic hernia? Comparison and metaanalysis of porcine small intestinal submucosa and polytetrafluoroethylene. J Pediatr Surg 2012; 47(8): 14961500, https://doi.org/10.1016/j.jpedsurg.2012.01.009.

5. Łukasiewicz A., Drewa T. Synthetic implants in hernia surgery. Adv Clin Exp Med 2014; 23(1): 135-142, https://doi. org/10.17219/acem/37037.

6. Ghanavati Z., Neisi N., Bayati V., Makvandi M. The influence of substrate topography and biomaterial substance on skin wound healing. Anat Cell Biol 2015; 48(4): 251-257, https://doi.org/10.5115/acb.2015.48.4.251.

7. Arutyunyan I.V., Tenchurin T.Kh., Kananykhina E.Y., Chernikov V.P., Vasyukova O.A., Elchaninov A.V., Makarov A.V., Korshunov A.A., Burov A.A., Podurovskaya Y.L., Chuprynin V.D., Uvarova E.V., Degtyarev D.N., Shepelev A.D., Mamagulashvili V.G., Kamyshinskiy R.A., Krasheninnikov S.V., Chvalun S.N., Fatkhudinov T.Kh. Nonwoven polycaprolactone scaffolds for tissue engineering: the choice of the structure and the method of cell seeding. Genes and Cells 2017; 12(1)1: 6271, https://doi.org/10.23868/201703009.

8. Zhao W., Ju Y.M., Christ G., Atala A., Yoo J.J., Lee S.J. Diaphragmatic muscle reconstruction with an aligned electrospun poly(E-caprolactone)/collagen hybrid scaffold. Biomaterials 2013; 34(33): 8235-8240, https://doi. org/10.1016/j.biomaterials.2013.07.057.

9. Pawar R.P., Tekale S.U., Shisodia S.U., Totre J.T., Domb A.J. Biomedical applications of poly(lactic acid). Recent Pat Regen Med 2014; 4(1): 40-51, https://doi.org/10.2174/221 0296504666140402235024.

10. Hanson S., D'Souza R.N., Hematti P. Biomaterialmesenchymal stem cell constructs for immunomodulation in composite tissue engineering. Tissue Eng Part A 2014; 20(1516): 2162-2168, https://doi.org/10.1089/ten.tea.2013.0359.

11. Baker S.R., Banerjee S., Bonin K., Guthold M. Determining the mechanical properties of electrospun poly- $\varepsilon-$ caprolactone (PCL) nanofibers using AFM and a novel fiber anchoring technique. Mater Sci Eng C Mater Biol Appl 2016; 59: 203-112, https://doi.org/10.1016/j.msec.2015.09.102.

12. Bagaeva V.V., Popova V.M., Pashkova G.S., 
Isadzhanyan K.E., Nikitin V.V., Zhilenkov E.L. The study the efficacy and safety of antimicrobial agents. Issledovaniya i praktika $v$ meditsine 2015; 2(3): 35-42, https://doi. org/10.17709/2409-2231-2015-2-3-35-42.

13. Yamanaka K., Yamamoto K., Sakai Y., Suda Y., Shigemitsu Y., Kaneko T., Kato K., Kumagai T., Kato Y. Seeding of mesenchymal stem cells into inner part of interconnected porous biodegradable scaffold by a new method with a filter paper. Dent Mater J 2015; 34(1): 78-85, https://doi. org/10.4012/dmj.2013-330.

14. Lv X.G., Feng C., Fu Q., Xie H., Wang Y., Huang J.W., Xie M.K., Atala A., Xu Y.M., Zhao W.X. Comparative study of different seeding methods based on a multilayer SIS scaffold: which is the optimal procedure for urethral tissue engineering? $J$ Biomed Mater Res B Appl Biomater 2016; 104(6): 10981108, https://doi.org/10.1002/jbm.b.33460.

15. Brouwer K.M., Daamen W.F., van Lochem N., Reijnen D., Wijnen R.M., van Kuppevelt T.H. Construction and in vivo evaluation of a dual layered collagenous scaffold with a radial pore structure for repair of the diaphragm. Acta
Biomater 2013; 9(6): 6844-6851, https://doi.org/10.1016/j. actbio.2013.03.003.

16. Urita Y., Komuro H., Chen G., Shinya M., Saihara R., Kaneko M. Evaluation of diaphragmatic hernia repair using PLGA mesh-collagen sponge hybrid scaffold: an experimental study in a rat model. Pediatr Surg Int 2008; 24(9): 1041-1045, https://doi.org/10.1007/s00383-008-2212-y.

17. Conconi M.T., De Coppi P., Bellini S., Zara G., Sabatti M., Marzaro M., Zanon G.F., Gamba P.G., Parnigotto P.P., Nussdorfer G.G. Homologous muscle acellular matrix seeded with autologous myoblasts as a tissue-engineering approach to abdominal wall-defect repair. Biomaterials 2005; 26(15): 2567-2574, https://doi. org/10.1016/j.biomaterials.2004.07.035.

18. Romanova O.A., Grigor'ev T.E., Goncharov M.E., Rudyak S.G., Solov'yova E.V., Krasheninnikov S.V., Saprykin V.P., Sytina E.V., Chvalun S.N., Pal'tsev M.A., Panteleev A.A. Chitosan as a modifying component of artificial scaffold for tissue engineering of human skin. Kletochnye tekhnologii v biologii i meditsine 2015; 2: 103-113. 